\title{
Article \\ Trajectory and Conveyance Validation of a Micro Conveyor Based on a Digital Electromagnetic Actuators Array for the Micro-Factory
}

\author{
Simon Duque Tisnes (D), Atif Tasneem, Laurent Petit*(D) and Christine Prelle $\mathbb{D}$ \\ Université de Technologie de Compiègne (UTC), Laboratoire Roberval (Mechanics, Energy and Electricity), \\ Centre de Recherche Royallieu CS 60319, 60203 Compiègne, France; saimonduque@gmail.com (S.D.T.); \\ atif.tasneem0@gmail.com (A.T.); christine.prelle@utc.fr (C.P.) \\ * Correspondence: laurent.petit@utc.fr
}

Citation: Duque Tisnes, S.; Tasneem, A.; Petit, L.; Prelle, C. Trajectory and Conveyance Validation of a Micro Conveyor Based on a Digital Electromagnetic Actuators Array for the Micro-Factory. Appl. Sci. 2021, 11, 11980. https://doi.org/10.3390/app 112411980

Academic Editor: Jose Machado

Received: 19 November 2021 Accepted: 14 December 2021 Published: 16 December 2021

Publisher's Note: MDPI stays neutral with regard to jurisdictional claims in published maps and institutional affiliations.

Copyright: (c) 2021 by the authors. Licensee MDPI, Basel, Switzerland. This article is an open access article distributed under the terms and conditions of the Creative Commons Attribution (CC BY) license (https:// creativecommons.org/licenses/by/ $4.0 /)$.
Abstract: Micro-factories are characterized by high modularity, reconfigurability and mobility. To achieve this, the micro-factory needs a conveyor which is able to transport objects in as many degrees of freedom (DoF) as possible, executes optimal trajectories of these objects in terms of energy and precision and is robust to withstand possible malfunctions. In this article, we present the planar conveyance of objects on a digital actuation array following trajectories generated by an adapted $\mathrm{A}^{*}$ algorithm. The $\mathrm{A}^{*}$ algorithm exploits the predictions of a developed dynamic model of the system to find the optimal paths (in terms of energy) on the conveyor surface. The dynamic model predictions were compared to experimental measurements, obtaining low root-mean-square-errors for all conditions. Uni-dimensional conveyance tests characterized the influence of the control parameters. Then, bi-dimensional motions characterized the conveyor's performance. From the bi-dimensional test, a position root-mean-square-error of $20 \mu \mathrm{m}$ was measured for a $1109 \mu \mathrm{m}$ open-loop controlled trajectory. The modular nature of the array allows easy scaling and avoiding possible malfunctioning zones, increasing the robustness of the micro-conveyor. The experimental tests demonstrate that the proposed device is an interesting alternative for the micro-factory.

Keywords: conveyance system; micro-factory; discrete actuator; collaborative actuation; MEMS array; digital actuation; electromagnetic actuator; planar motion

\section{Introduction}

\subsection{Context}

The increased demand for compact, intelligent and multi-functional products can only be met by adding more sensors, actuators and processors into the product. Miniaturization has been the industry's answer to this new paradigm of market demand by downscaling the components of the product to increase their density [1]. Miniaturized components have an impact on the manufacturing systems, requiring specialized machinery, techniques and processes to produce these small components. To solve this impact on the manufacturing system, micro-factories are a proposed solution [2-5]. Micro-factories are defined as smallsize production systems suitable for the manufacture of components with dimensions close to the size of the produced goods. Micro-factories are characterized by high modularity, reconfigurability and mobility. To obtain these characteristics, the micro-factory needs to properly sort, convey and align all materials in each production step. These tasks, usually requiring micrometric precision, are achieved by the conveyor of such a micro-factory (thus micro-conveyor), making it a key element of the system.

\subsection{Micro-Conveyor Examples}

Different physical principles have been used to obtain planar motion micro-conveyors such as: electrostatic [6], electromagnetic [7,8], piezoelectric [9] and pneumatic [10-12]. Air 
jet pneumatic conveyors generate fast and contactless movements: in [10], the authors obtained speeds of $300 \mathrm{~mm} \mathrm{~s}^{-1}$ with precision of $93 \mu \mathrm{m}$. Soft actuated pneumatic conveyors generate medium forces with contact movements: in [12] the authors obtained $70 \mathrm{~mm} \mathrm{~s}^{-1}$ movements with 16 degrees of freedom (DoF). On the other hand, all pneumatic conveyors require constant energy input to hold a position, high pressures, complex fluid models and control. They are also difficult to integrate due to their pipework. Piezoelectric conveyors achieve long-range motions with sub-micrometer accuracy: the system presented in [9] had a range of $10 \mathrm{~mm}$ with a resolution of $2 \mu \mathrm{m}$ and speed of $0.3 \mathrm{~mm} \mathrm{~s}^{-1}$. On the other hand, they need high working voltages at high frequencies, are sensible to temperature and present strong nonlinearities, requiring complex control. Electromagnetic systems present large strokes with medium forces, slower response times than piezoelectric or electrostatic actuators but faster than electrothermal actuators, are relatively easy to build and assemble, and could generate levitation and driving forces. In [8], a Maglev conveyor achieved speeds of $2.3 \mathrm{~mm} \mathrm{~s}^{-1}$ with resolutions of $100 \mu \mathrm{m}$. Nevertheless, they do not scale as well as the other systems beyond the millimeter size.

\subsection{From Analog to Digital Actuation}

Planar motion could be based on continuous (analog) actuators, able to attain and keep any position inside the actuator stroke. These actuators present high performances and are generally controlled in closed-loop with high-precision sensors, making their integration in compact systems complex. Another architecture based on a collaboration of digital actuators could also be used for planar motions. The stroke of digital actuators is divided into well-defined, repeatable and stable positions that enables sensorless solutions. Once the mobile part reaches one of the discrete position, the stability of the position maintains the mobile part in place. Energy is only needed to switch the mobile part between the discrete positions, enabling the use of a simple pulsed control signal. Due to the digital behavior, an open loop control can be used, simplifying the integration of digital actuators in compact or highly integrated systems.

In the literature, digital actuators are used as switches or arranged in a collaborative actuation array for more complex tasks. Different physical principles have been used to create digital actuators. The authors in [13] used an electromagnetic bistable actuator as a micro switch, with $160 \mu \mathrm{m}$ stroke and a closing time of $0.96 \mathrm{~s}$. In [14], the authors propose a chain of two DoF digital electromagnetic tilting actuators to perform complex movements and manipulations as a modular robot chain. The authors in [15] built a $50 \times 50$ optical switch matrix using digital electrostatic actuators to guide the light. As their strokes were $1 \mu \mathrm{m}$, they obtained switching times of $0.85 \mu \mathrm{s}$. The authors in [16] proposed a tactile display with an elastomer actuator working with an electrostatic actuator producing digital actuation. The resulting actuator had a stroke of $680 \mu \mathrm{m}$ and $185 \mathrm{mN}$ blocking force. A combination of electromagnetic and piezoelectric principles for multistable digital actuation was presented in [17]. The actuator consists of permanent magnetic rings, defining multiple stable positions: a lower one in contact with a piezoelectric stack actuator, and upper ones located at predefined heights, where the magnetic object is stably levitated. The piezo stack actuator provides a fast initial acceleration of the magnet, improving performance, and then solenoids control which final position is attained. In [18], the authors fabricated a DAC system based on a multistable electrostactic actuator array. Their system showed a total stroke of $150 \mu \mathrm{m}$ with eight defined positions at a control voltage of $60 \mathrm{~V}$. The minimum switching time was $0.1 \mathrm{~ms}$. The authors in [19] presented a shape memory alloy actuated anti-slip mechanism for the wheels of step climbing robots. The proposed mechanism used a bi-stable four-bar mechanism to clamp on the edges of the stairs to avoid slipping, blocking the wheel of the robot while activated and passively returning to the closed position when not needed.

In addition to the presented applications, digital actuators have been used for planar conveyance devices. In [20], the authors presented a $20 \times 20$ array of ciliary electrothermal digital actuators. The conveyor had a minimum step of 2 to $18 \mu \mathrm{m}$ and a maximum 
conveyance speed of $0.65 \mathrm{~mm} \mathrm{~s}^{-1}$. In [21], the author developed a $4 \times 4$ array of digital electrothermal actuators as a micro-conveyor based on lift and slide principle. Each actuator had a force of $80 \mu \mathrm{N}$ and a stroke of $3 \mu \mathrm{m}$. The minimum step size was $0.28 \mu \mathrm{m}$, with a maximum speed of $56.8 \mu \mathrm{m} \mathrm{s}^{-1}$. Both systems in $[20,21]$ are electrothermal, known to generate large strokes and deflections with important forces, but have slow response times (thus slow conveyance speeds) and consume more power than other actuation principles.

Planar motion conveyors based on digital actuators produce a step by step output which can be limiting if a smooth continuous motion is required. Nevertheless, collaboration of digital actuators for planar motion presents some advantages: besides simple integration and open-loop control, this kind of systems is easily scalable to be adapted to the application needs. In case of disturbances, the digital principle ensures the position holding without energy supply and in case of failures of one of several digital actuators, the overall working is preserved in a robust manner. Performances of collaborative digital systems require high manufacturing precision because the effect of manufacturing errors can not be compensated by the control. Microfabrication is then often used to manufacture these systems, particularly batch fabrication in case of devices based on several digital actuators.

\subsection{Contribution of This Article}

This article proposes a planar micro-conveyor based on a digital electromagnetic actuator array. To execute the conveyance task, we solve the pathfinding problem with a grid representation of the conveyor and a trajectory algorithm based on the $\mathrm{A}^{*}$ algorithm. The resulting trajectory is sent to a developed dynamic model of the conveyor that finds the optimal control variables to execute this trajectory. Therefore, the micro-conveyor asks a list of wished positions to attain, and the possible obstacles to avoid in the environment, and it plans and executes an energy optimal trajectory between these points, avoiding collisions with the obstacles.

We present the execution and characterization of bi-dimensional trajectories on the proposed micro-conveyor. As performance metrics, we find: a straightness error of $2.55 \mu \mathrm{m}$ for uni-dimensional trajectories and an average displacement root-mean-square-error of $19.8 \mu \mathrm{m}$ for open-loop controlled planar trajectories.

This article is organized as follows: we first present the principle of the conveyor in Section 2. The dynamic model of the system is described in Section 3. Then, Section 4 gives the materials and methods for the experimental tests. Then, we characterize the performance of the conveyor and analyze the results in Section 5. Finally, we synthesize our conclusions and the performance of the proposed micro-conveyor against the literature alternatives in Section 6.

\section{Principle of the Conveyance System}

\subsection{Principle of the Elementary Digital Actuator (EDA)}

The basic component of the micro conveyor is the Elemental Digital Actuator (EDA). The EDA is a silicon structure with five NdFeB Permanent Magnets (PMs) as shown in Figure 1. The mobile part of the actuator is the central PM, called Mobile Permanent Magnet (MPM), with dimensions $2 \mathrm{~mm} \times 2 \mathrm{~mm} \times 1.2 \mathrm{~mm}$. The MPM lies in a slightly larger square cavity on the silicon (dimensions $2.2 \mathrm{~mm} \times 2.2 \mathrm{~mm} \times 1 \mathrm{~mm}$ ). The stroke of the EDA in both $x$ and $y$ directions is defined by this gap between the MPM and the cavity $(0.2 \mathrm{~mm})$. The other four surrounding PMs are fixed in the silicon structure, thus named Fixed Permanent Magnets (FPMs). Each FPM is located at $2.6 \mathrm{~mm}$ from the MPM side $\left(\mathrm{d}_{1}\right.$ in Figure 1). The magnetization of the MPM and FPMs are directed along the $z$ axis in opposite directions to obtain an attracting magnetic force between them. This configuration creates four equilibrium positions for the MPM at each corner of the cavity, giving a digital actuation nature to the EDA.

Two pairs of wires under the cavity are used to actuate the MPM along the $x$ and $y$ axes. The principle of actuation of the EDA is the Lorentz's force generated by the current carried by these wires and the magnetic field of the MPM. The current used to displace the 
MPM is called "driving current" $\left(\mathrm{I}_{\mathrm{d}}\right)$. We called the wires by its actuating axis, i.e., the $x$ wires are the ones actuating the MPM in the $x$-axis as shown Figure 1. Each pair of wires is printed in different planes of a multi-layer PCB circuit, in order to avoid electrical contact between them. Both actuating wires can operate simultaneously. If both pairs of wires are activated, the MPM will move in a diagonal direction, and both currents are considered as driving currents. If one current is used to displace the MPM and the other generates a force towards the cavity wall, this last current is called "holding current". The holding current could be used to reduce the straightness error of the MPM's displacement along the moving axis, as it forces the MPM to stay in contact with the straight silicon wall. Finally, a thin glass layer is placed between the MPM and the PCB to avoid their direct contact and give the MPM a plane surface to slide between the discrete positions.

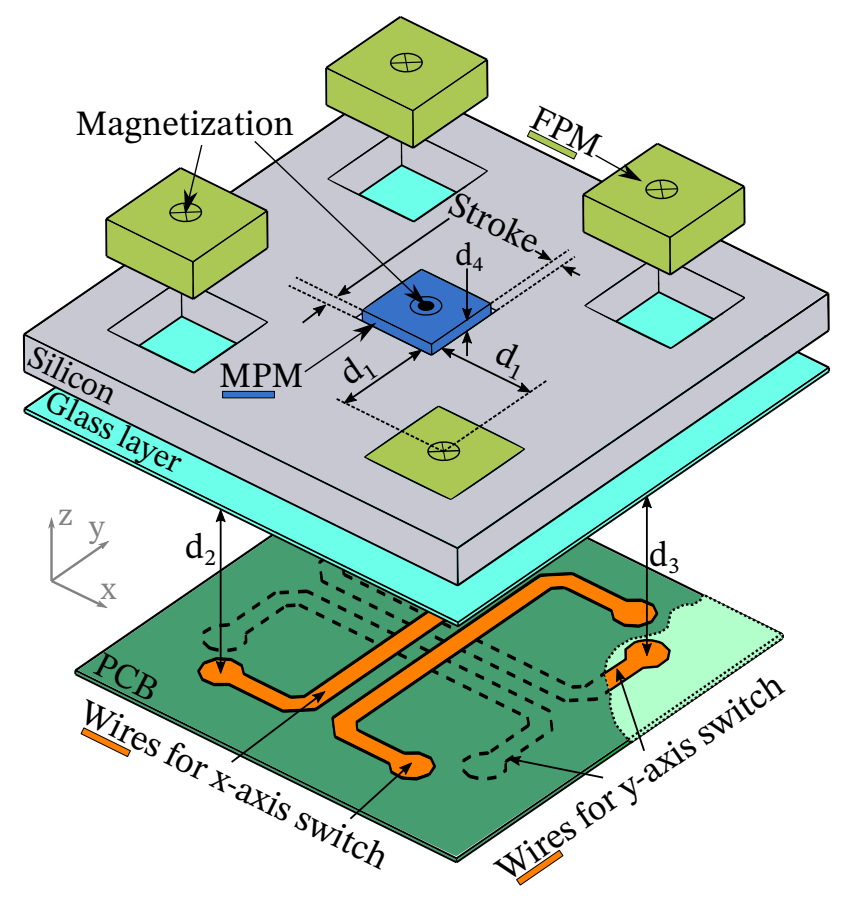

Figure 1. Elementary Digital Actuator (EDA).

\subsection{Principle of the Digital Actuator Array}

The micro conveyor is formed with an array of EDAs: a Digital Actuator Array (DAA). Adjacent EDAs share a pair of FPMs to decrease the DAA size (Figure 2). The conveyed object is placed on a glass tray that contacts the top side of the MPMs. The DAA combines individual actions of each EDA to obtain a cumulative displacement of the conveyed object based on a "stick-slip" principle. The strategy to convey an object on top of the DAA using this collaborative actuation is presented in Figure 3. The conveyed object is placed on top of the MPMs (a). The object is considered to be non-ferromagnetic to avoid magnetic interactions with the actuators. The EDAs are simultaneously switched to displace the object (b). During this step, the friction force between the MPMs and the conveyed object accelerates the object until the MPMs reach their discrete position (stick phase). Once the MPMs stop, the dynamic friction due to the relative movement between the moving object and the static MPMs acts as a brake to the object until it stops, reaching a "displacement step". The second phase "slip" resets the configuration for a new displacement step: each MPM is individually switched back to return to its initial position ((c) to (d)). The friction force between a single MPM and the object is lower than the opposition force of the other MPMs. During steps (c) and (d), the MPM slides relatively to the object which, in theory, does not generate motion. In practice, a small "return displacement" was observed due to non-uniform distribution of the object weight on the MPM and friction inhomogeneity. This influence will be studied in the experimental section. When all the MPMs are back to 
the initial position, a new step of displacement of the object can be done (a). The current used to return the MPMs to the initial position is noted as return current $\left(I_{r}\right)$ in Figure 3. The DAA can be extended in a modular way by adding EDAs to the array .
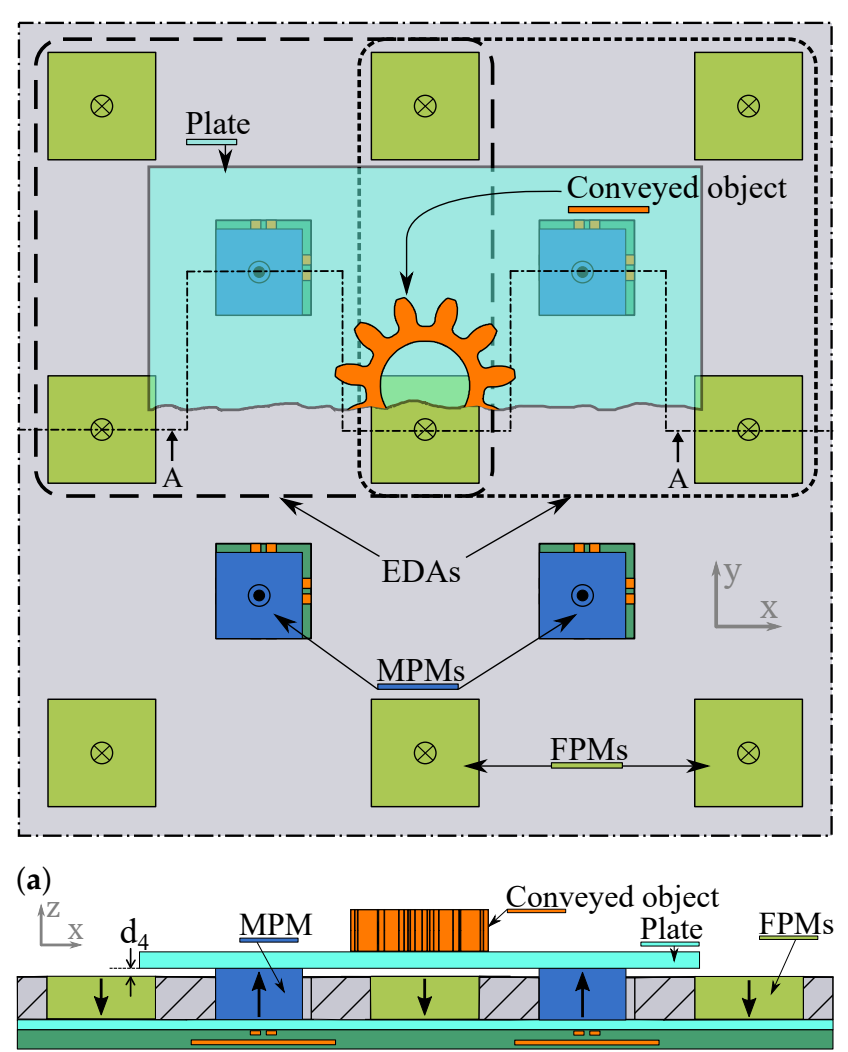

(b)

Figure 2. $2 \times 2$ Digital Actuator Array (DAA). (a) Top view. (b) Front view (A-A cut).

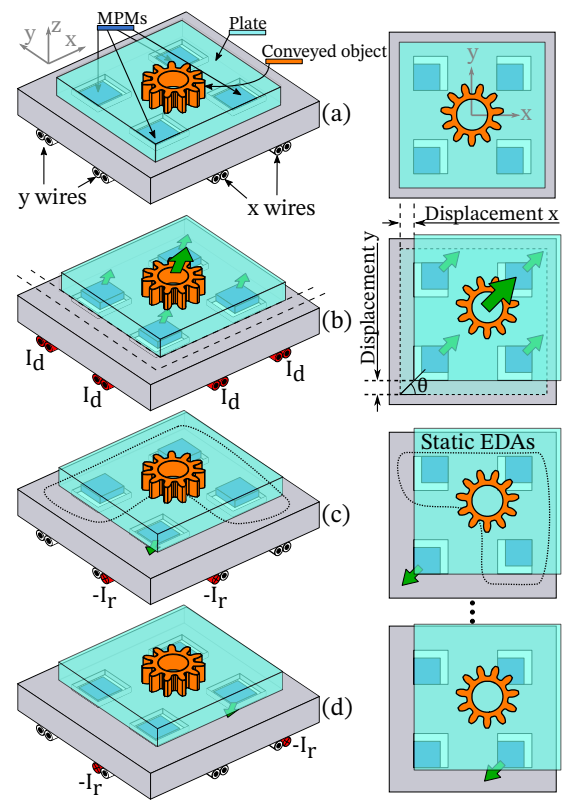

Figure 3. Side (left) and Top (right) view of the conveyance principle of the DAA. A collaborative effort of the MPMs and individual return of each one (one displacement step represented).

\section{Dynamic Model}

To control the DAA in open loop, we developed a dynamic model that predicts the conveyed object displacement as a function of the currents in the actuating wires. 
The model considers the magnetic forces $\left(\vec{F}_{m}\right)$ due to the interaction between the PMs, electromagnetic forces $\left(\vec{F}_{e m}\right)$ due to the interaction between the PMs and the currents in the $\mathrm{x}$ and $\mathrm{y}$ wires, and friction forces $\left(\vec{F}_{f 1, f 2, f_{3}}\right)$ due to the contact between moving parts (MPM with surroundings). We developed and validated this dynamic model on a previous work [22]. In this section, we briefly explain the dynamic model and the information flow to predict the conveyed object displacement.

Figure 4 presents the free body diagram of the EDA. Once the total force onto the MPM is calculated, we process the dynamic effects of collisions and rebounds of the MPM as it reaches the stroke limit. Finally, the DAA is considered as a perfectly homogeneous array, with each MPM contributing an equal amount of force to the conveyed object.

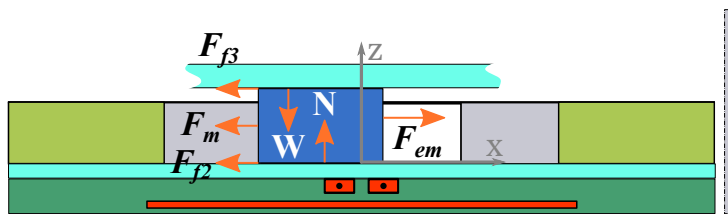

(a)

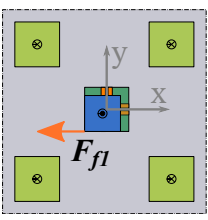

(b)

Figure 4. EDA's free body diagram (a) Side view. (b) Top view.

\subsection{Magnetic Forces Modeling}

We use the Gilbert charge model to compute the magnetic force exerted on one PM due to another PM magnetic flux density [23]. We consider the magnetization of the PMs as $\left(\mathbf{M}=M_{s} \mathbf{z}\right.$, Table 1). To automate the calculation in a computer, we divide each PM surface $S$ into $p$ areas $\Delta A$ to obtain a semi-analytic and discrete version of magnetic force (Equation (1)).

$$
\mathbf{F}_{\text {magnetic }}=\sum_{p} \sigma_{m}\left(x_{p}\right) \mathbf{B}_{\mathrm{ext}}\left(x_{p}\right) \Delta A_{p}
$$

With $\sigma_{m}$ the surface current density of the PMs and $\mathbf{B}_{\text {ext }}$ the total external magnetic flux density generated by the first PM onto the second PM. $\mathbf{B}_{\text {ext }}$ is derived from the charge model considering each PM as a perfect square prism [23].

Table 1. EDA characteristics.

\begin{tabular}{ccccc}
\hline Element & Dimensions $(\mathbf{m m})$ & Mass & Material & Mag. (T) \\
\hline MPM & $2.0 \times 2.0 \times 1.2$ & $34 \mathrm{mg}$ & $\mathrm{NdFeB}$ & 1.22 \\
FPM & $2.2 \times 2.2 \times 1.0$ & $45 \mathrm{mg}$ & $\mathrm{NdFeB}$ & 1.43 \\
Structure & $9.9 \times 9.9 \times 1.0$ & $104 \mathrm{~g}$ & $\mathrm{Si}$ & - \\
Glass layer & $4.0 \times 4.0 \times 0.1$ & $64 \mathrm{mg}$ & Glass & - \\
Object & $20 \times 20 \times 0.1$ & $416 \mathrm{mg}$ & Glass & - \\
\hline \multicolumn{7}{c}{ Distances $(\mathrm{mm})$} & & \\
\hline $\mathrm{d}_{1}=2.6$ & $\mathrm{~d}_{2}=0.185$ & $\mathrm{~d}_{3}=0.253$ & $\mathrm{~d}_{4}=0.2$ & Stroke $=0.2$ \\
\hline
\end{tabular}

\subsection{Electromagnetic Force Modeling}

The force exerted on the MPM by the current-carrying wires beneath it is modeled with the Lorentz force equation. The current-carrying wires are considered as thin filaments, obtaining Equation (2), with $I$ the current intensity through the wire and d ll the vector along the wire length and $\mathbf{B}_{\text {ext }}$ the external magnetic flux density field.

$$
\mathbf{F}_{\text {electromagnetic }}=I \int_{\text {wire }} \mathrm{d} \mathbf{l} \times \mathbf{B}_{\text {ext }}
$$

This expression models the driving, holding and return currents interactions with the MPM. 


\subsection{Friction Modeling}

The displacement of the object on the DAA is based on a "stick-slip" frictional principle. To model all friction phenomena involving the MPM the work of Bengisu and Akay [24] was adapted (Equation (3)). Their model captures the Stribeck effect that was experimentally observed in our system [25].

$$
\mathbf{F}_{\text {friction }}=\begin{array}{ll}
\left(-\frac{F_{s}}{v_{0}^{2}}\left(\|v s \cdot\|-v_{0}\right)^{2}+F_{s}\right) \operatorname{sgn}(v) & \|v\|<v_{0} \\
\left(F_{c}+\left(F_{s}-F_{c}\right) e^{-\zeta\left(\|v s .\|-v_{0}\right)}\right) \operatorname{sgn}(v) & \|v\|>v_{0}
\end{array}
$$

With $v$ the speed of the MPM, $F_{s}$ the value of static friction, $F_{c}$ the value of the settling friction when $v \rightarrow \infty, v_{0}$ the transition speed value between the static friction and dynamic friction and $\zeta$ the decay factor for the dynamic friction. The values of these parameters were experimentally determined.

\subsection{Collision and Rebound Effect Modeling}

The MPM impacts the cavity walls when it arrives to its discrete positions. The collision and rebound of the MPM due to this impact were modeled implementing the classic equations of elasto-plastic collisions and considering the silicon structure massive enough to be static upon impact of the MPM [26] (Equation (4)).

$$
v_{\mathrm{a}}=\frac{m_{\mathrm{a}} u_{\mathrm{a}}+m_{\mathrm{b}} u_{\mathrm{b}}+m_{\mathrm{b}} c_{r}\left(u_{\mathrm{b}}-u_{\mathrm{a}}\right)}{m_{\mathrm{a}}+m_{\mathrm{b}}}
$$

With $v_{\mathrm{a}}$ and $u_{\mathrm{a}}$ the speed of the MPM after and before collision, respectively, and $u_{\mathrm{b}}$ the speed of the structure before collision. $m_{\mathrm{a}}$ and $m_{\mathrm{b}}$ the mass of the MPM and structure, respectively. We suppose that the structure is static before and after the collision $\left(u_{\mathrm{b}}=0\right)$ given its large mass compared to the one of the MPM $\left(m_{\mathrm{b}} \gg m_{\mathrm{a}}\right)$.

The coefficient of restitution $\left(c_{r}\right)$ for the collision between the MPM and the structure is modeled following the work of Weir and Tallon for elasto-plastic impacts [27]:

$$
c_{r}=\alpha\left(\frac{Y}{1}\right)^{5 / 8}\left(\frac{1}{E_{*}}\right)^{1 / 2}\left(\frac{R_{1}}{R_{*}}\right)^{3 / 8}\left(\frac{1}{v}\right)^{1 / 4}\left(\frac{1}{\rho}\right)^{1 / 8}
$$

With $v$ the impact speed, $Y$ yield pressure, $E_{*}$ the equivalent Young modulus and $\rho$ the density of the material in collision (in our case $\mathrm{NdFeB}$ into silicon). The term $R_{1} / R_{*}$ arises because of the different possible geometries after separation (and penetration) depending on the materials' hardness and shape. For impacts in which either surface is plane, or both surfaces suffer plastic deformation, the ratio $R_{1} / R$ is neglected [27]. Finally, $\alpha$ is a coefficient dependent of the impact theoretical shape and energy transmission. Weir and Tallon worked with sphere-sphere impacts, deducing $\alpha=3.1$. The collision between the MPM and structure is a plane-plane impact, resulting in $\alpha=1$ [27].

\subsection{Dynamic Model Flowchart}

Figure 5 represents the flowchart of the developed dynamic model. Based on the EDA and DAA characteristics, the magnetic, electromagnetic and friction forces are computed for a given time step. Then, Newton's second law is used to determine the MPM and conveyed object accelerations. The obtained accelerations are then integrated to obtain the speeds and displacements of the MPM and of the conveyed object considering the collision and rebound effects. Then, the calculation for the next time step can be done. 


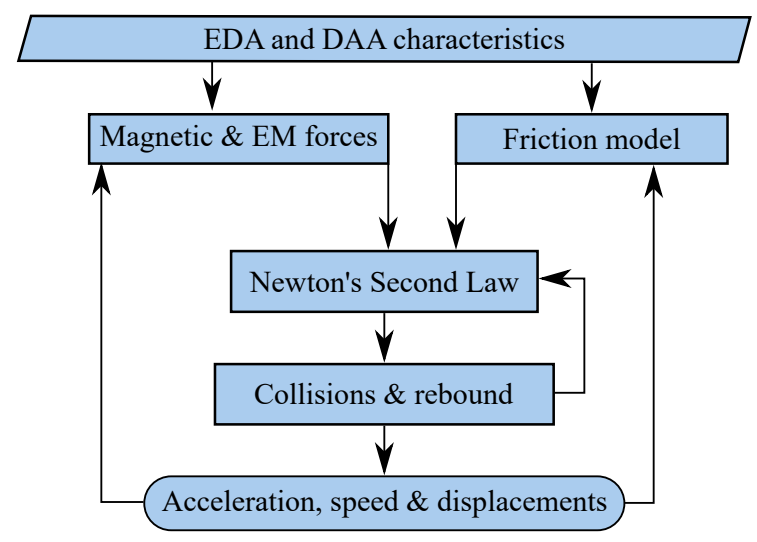

Figure 5. Flowchart of the dynamic model.

\section{Materials}

\subsection{DAA Prototype}

Figure 6 shows a picture of the prototyped DAA. The system is controlled with a LabVIEW interface, connected to a National Instruments PCI6733 input/output board that sends the control voltages $( \pm 10 \mathrm{~V})$ to four current sources, producing a maximal output of $\pm 10 \mathrm{~A}$ each. These generated currents are the control variables of the system and are injected into the DAA. The control signals of the DAA are constant-valued current pulses that are maintained for $20 \mathrm{~ms}$. This pulse duration was selected to be longer than the dynamics of the DAA (in the order of 5-15 ms). Figure 7 represents the current signal used during experimentation. The first current pulse, indicated as "driving and holding currents", ensures the plate motion (as shown in Figure 2b) and the second one, indicated as "return current", ensures the return of the MPM to its initial position (as shown Figure 2c). The influences of the driving, holding and return currents on the device behavior were studied and are presented in Section 5. The total displacement of the object is measured using a camera on top of the DAA, a Grasshopper3 GS3-U3-32S4C with a fixed optic system, providing an image with a $0.694 \mu \mathrm{m} /$ pixel resolution. The conveyed object was a glass plate with a sticker target. The object weight was $416 \pm 1 \mathrm{mg}$ and had a size of $22 \mathrm{~mm} \times 22 \mathrm{~mm} \times 0.13 \mathrm{~mm}$. A movie showing the MPM switching (driving current of 3.5A) and the plate displacement (two steps) along $x$-axis is available in a supplementary file.

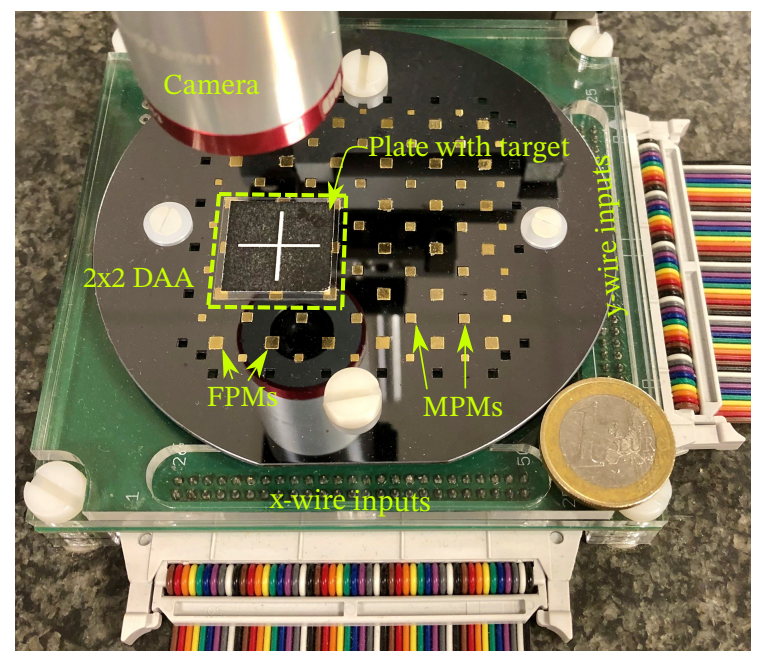

Figure 6. Picture of the real implementation and components. 


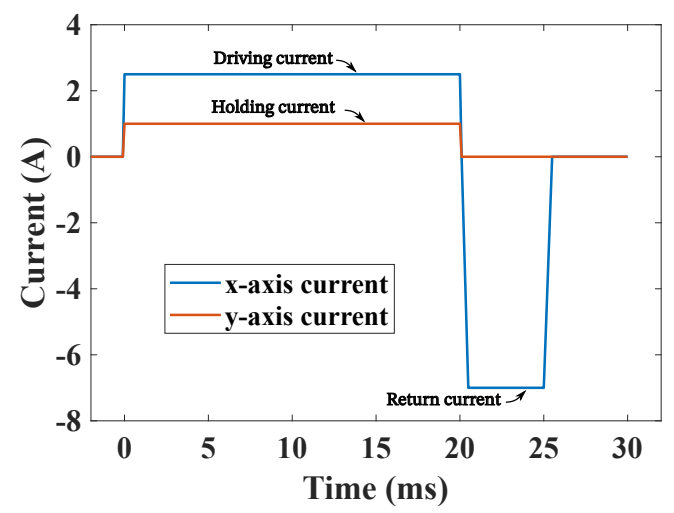

Figure 7. Representation of the current signals used during experimentation.

\subsection{Trajectory Algorithm}

The DAA produces displacement steps, thus the displacement evolution in space is well represented by a discrete state-space, or a grid. Therefore, grid-based pathfinding algorithms are a good choice for our conveyance task. Consequently, an $\mathrm{A}^{*}$ trajectory algorithm, based on the presented dynamic model, was developed. Its objective is to transport the object to any succession of target points, avoiding the possible obstacles and maximizing the performance of the system. To solve the conveyance trajectory, we suppose to know the initial position of the object's center, the list of target points and the obstacles coordinates. First, we divide the conveyance plane into a grid representing the possible positions and transitions of the object on the DAA. The target positions and obstacles are integrated into the grid, and then the grid is injected into the trajectory algorithm to find the succession of displacement steps that link all target points. The distance and direction of each step is then sent to the dynamic model, that defines the control currents that produce the desired displacement steps, minimizing the current intensities. Finally, these control currents are sent to a virtual system as well as to the real system simultaneously to visualize and evaluate the generated displacements (Figure 8).

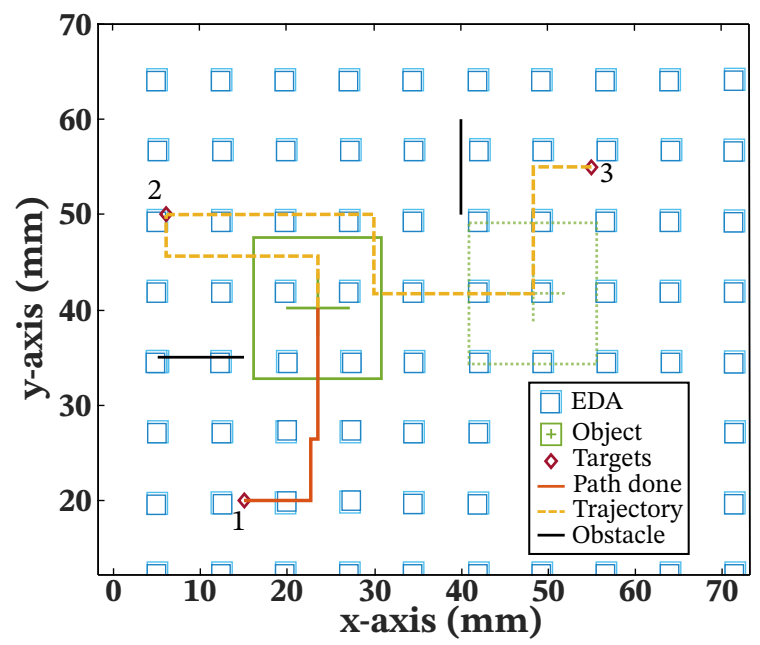

Figure 8. Interface: A pathfinding problem with 3 target points and two obstacles solved with the adapted $\mathrm{A}^{*}$ algorithm and dynamic model (and a $1 \mathrm{~mm}$ safety distance to the obstacles).

Our previous work demonstrated that an optimum control strategy in terms of energy and final position error was to use the maximum axial displacement at each step to approach the target point and then use a correction step at the end of the trajectory to attain the final position [28]. The $\mathrm{A}^{*}$ algorithm uses this control strategy, generating a grid that allows displacements only in the axial directions. Each node of the grid is at the maximum displacement step distance from each other. The target points are approximated 
to the nearest node and, once the object arrives there, the difference to the real target is then injected into the inverse model to find the correction step. As the $\mathrm{A}^{*}$ algorithm minimizes the distance to the obstacles in its search for the optimum trajectory, we included an additional parameter, called safety margin, to force the path to respect a distance to the obstacles. This margin is set to $1 \mathrm{~mm}$ in the example of Figure 8 .

\section{Methods, Results and Discussion}

In this section, we compare experimental and simulated results of the DAA conveying a glass layer as object. We first validate the model prediction of the driving and holding currents influence on a displacement step. Then, we validate the principle of conveyance of the DAA and measure the unwanted return displacement. Afterwards, we show the influence of the holding current on the straightness of the obtained trajectories. Afterwards, we detail the theoretical-experimental comparison of two bi-dimensional trajectories on the DAA to evaluate its open loop control performance.

\subsection{Driving and Holding Current Influence on a Displacement Step}

Using the model described in Section 3, we simulated the object displacement for different driving currents along $x$ and $y$-axes without and with a $1 \mathrm{~A}$ holding current. We injected a driving current in one of the two pair of wires of each actuator and, simultaneously, a holding current into the other pair of wires, to obtain a linear motion of the mobile permanent magnet (MPM) and the object along that axis. Both current pulses were longer than the displacement step time to ensure that the MPM accelerated throughout its displacement. Figure 9 presents the displacement of the conveyed object as a function of the driving current intensity for both the $x$ and $y$ axes with and without a 1 A holding current. Each experimental point in Figure 9 was measured 20 times under the same conditions to obtain a mean value and a standard deviation for the point's conditions. The figure shows the point as the mean value, and its standard deviation as error bars.

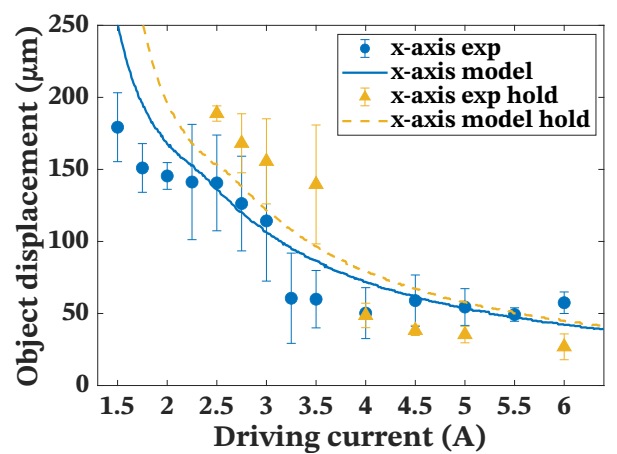

(a)

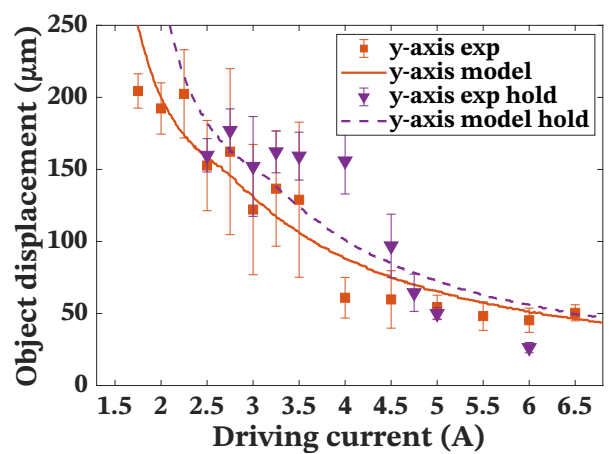

(b)

Figure 9. Driving and holding current influence on a displacement step: Object displacement vs. a varying driving current with and without a 1 A holding current. (a) $x$-axis step. (b) $y$-axis step.

From Figure 9, the object displacement decreased as the driving current increased on both axes and for both conditions (with and without the $1 \mathrm{~A}$ holding current). When the injected current increases, the MPM displacement time and the energy transfer through friction to the object are reduced, which leads to a decrease of the object displacement. For the same driving current value, the obtained displacement is lower along the $x$-axis than along the $y$-axis. This is due to the lower distance between the wires actuating the MPM along the $x$-axis than for the $y$-axis $\left(d_{2}\right.$ and $d_{3}$ in Table 1$)$. Therefore, the electromagnetic force is higher for the $x$-axis, inducing a shorter MPM displacement time and consequently a lower energy transfer to the object, as explained before. For example, with a $2 \mathrm{~A}$ driving current (without holding current), the object displacement along $x$ and $y$-axis are $151 \mu \mathrm{m}$ and $192.3 \mu \mathrm{m}$, respectively. The holding and no holding results followed a similar tendency. The holding current induced a shift towards higher driving currents (shift of the initial 
curve to the right). This is due to the higher driving current intensity needed to overcome the increased friction force generated by the holding current. The error bars decreased as the driving current increased for both conditions. This is attributed to the important influence of the friction conditions at lower currents and its reduced influence with faster kinematics as the current increases.

The model predicted the experimental tendency correctly. The root-mean-square error (RMSE) between the experimental and simulated results without holding current was $29.9 \mu \mathrm{m}$ for the $x$-axis and $19 \mu \mathrm{m}$ for the $y$-axis. The RMSE between the model prediction and experimental results with 1 A holding current were $38.6 \mu \mathrm{m}$ ) for the $x$-axis and $49.3 \mu \mathrm{m}$ for the $y$-axis. In order to implement our optimum control strategy, the minimum current values ensuring a motion were experimentally sought and found to be $1.5 \mathrm{~A}$ (corresponding displacement: $179 \mu \mathrm{m})$ and $1.75 \mathrm{~A}(205 \mu \mathrm{m})$ for $x$ and $y$-axes without holding current, respectively. When using a $1 \mathrm{~A}$ holding current, the minimum driving currents to ensure a motion were higher and found to be $2.5 \mathrm{~A}(189 \mu \mathrm{m})$ and $2.75 \mathrm{~A}(177 \mu \mathrm{m})$ for $x$ and $y$ axes, respectively.

The straightness error of all the experimental points of Figure 9 was measured. This error is attributed to the asynchronous movement of the MPMs due to the heterogeneity in friction, causing a torque to appear. Furthermore, manufacturing and assembly errors like the misalignment between the wires and the actuators' axes could contribute to this error. Figure 10 presents the straightness error measurement of the results presented in Figure 9.

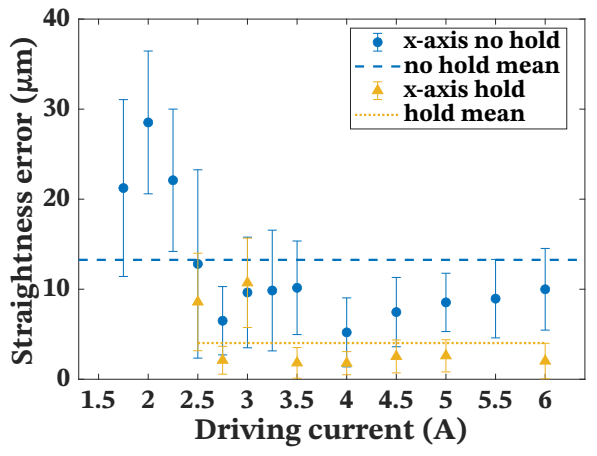

(a)

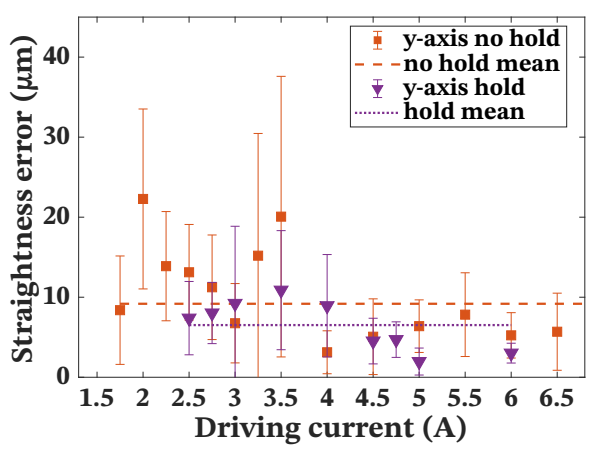

(b)

Figure 10. Holding current influence on straightness error. (a) $x$-axis results. (b) $y$-axis results.

From Figure 10, the straightness error decreased as the driving current increased for both axes and for both conditions (with and without holding current).

From Figure 10a ( $x$-axis), the mean straightness error without holding current was $13.3 \mu \mathrm{m}$, and it decreased to $4 \mu \mathrm{m}$ with $1 \mathrm{~A}$ holding current. From Figure 10b (y-axis), the mean straightness error without holding current was $9.1 \mu \mathrm{m}$, and it decreased to $6.5 \mu \mathrm{m}$ with $1 \mathrm{~A}$ holding current. These results show that the holding current reduced the straightness error, thanks to the use of the EDA silicon cavity wall (fabricated with microfabrication techniques) as a straight guide for the MPM movement.

From Figures 9 and 10, we can see that high currents are useful for small, repeatable and precise object displacement steps given the low standard deviation and straightness error. For example, injecting $6 \mathrm{~A}$ driving current and $1 \mathrm{~A}$ holding current generates a displacement step of: $26.86 \mu \mathrm{m}$ with a straightness error of $2 \mu \mathrm{m}$ in the $x$-axis, and $26.29 \mu \mathrm{m}$ with a straightness error of $3 \mu \mathrm{m}$ in the $y$-axis. The presented results confirm the interest of the proposed control strategy based on long displacement steps with low driving current values to achieve a high conveyance speed with low-energy consumption and finally a fine and adjustable step at the end of the trajectory with a higher driving current value. 


\subsection{Conveyance Validation and Return Step Influence}

Figure 11 shows a sequence of pictures at each conveyance step (explained in Figure 3). The displacement step is clearly visualized in Figure $11 \mathrm{~b}$ once the MPMs have moved. Then the return step impact on the object position is shown in Figure 11c.

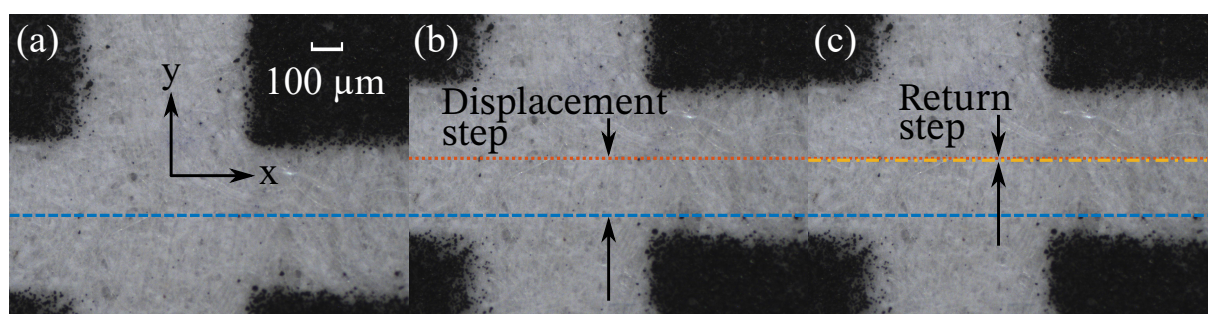

Figure 11. Object to be conveyed on the DAA (a), displacement step measure (b) and return step influence (c).

The trajectory controller assumes that the return step (the MPM reset process once a displacement step has been done) does not displace the object. In practice, a return displacement was observed, attributed to possible uneven distribution of the conveyed object weight on the MPMs and heterogeneity in friction conditions. The return current intensity influence on this return step was studied to find the best operating conditions for multi-step execution.

Different return current intensities along the $x$ and $y$ axes of a $2 \times 2$ DAA were injected following the return process. The DAA carried a $0.416 \mathrm{~g}$ object. Each return current intensity was executed 20 times to obtain a mean and standard deviation values. Figure 12 presents the return step displacement vs. return current intensity. From Figure 12, different behaviors were observed for the $x$-axis and $y$-axis.

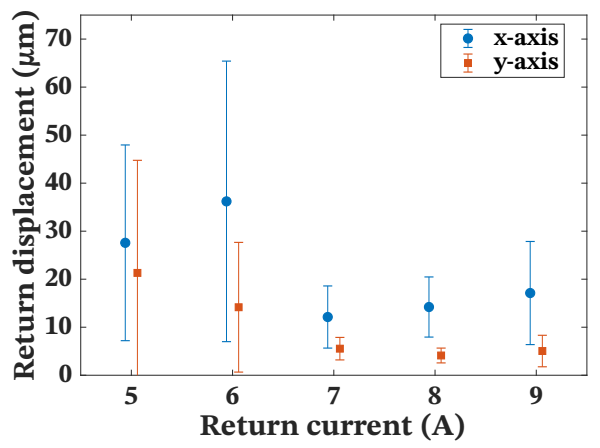

Figure 12. Return current influence on return displacement.

For the $y$-axis, the return displacement and its standard deviation decreased as the current intensity increased, until $8 \mathrm{~A}$. For the $9 \mathrm{~A}$ point, both return displacement and standard deviation increased from the previous experimental point. For the $x$-axis, the return displacement and its standard deviation increased when the return current intensity went from 5 to $6 \mathrm{~A}$. Then, the return displacement and its standard deviation collapsed to a minimum at $7 \mathrm{~A}$. Increasing the return current intensity further than $7 \mathrm{~A}$ resulted in a steady increase in return displacement and standard deviation. This behavior is attributed to the $z$ component of the electromagnetic force. For such high intensity currents, this force component overcomes the MPM and conveyed object's weight, making the MPM to "jump" inside its cavity. This jump produces a highly variable movement of the object along the magnet's displacement axis. The return current is then fixed at $7 \mathrm{~A}$ for both axes to reduce its influence on the conveyance task. 


\subsection{Holding Current Influence on Conveyance Trajectory}

Next, we explored the holding current influence on multiple displacement steps for straight trajectory generation. This was important as the trajectory algorithm was designed to execute uni-dimensional (axial) displacement steps and we are in an open loop control scheme. We executed 6 consecutive displacement steps in the $+x$-axis with and without a $1 \mathrm{~A}$ holding current and measured the deviation along the $y$-axis. This axial trajectory was executed 10 times to obtain a position standard deviation, represented by the error bars in Figure 13, that shows the results.

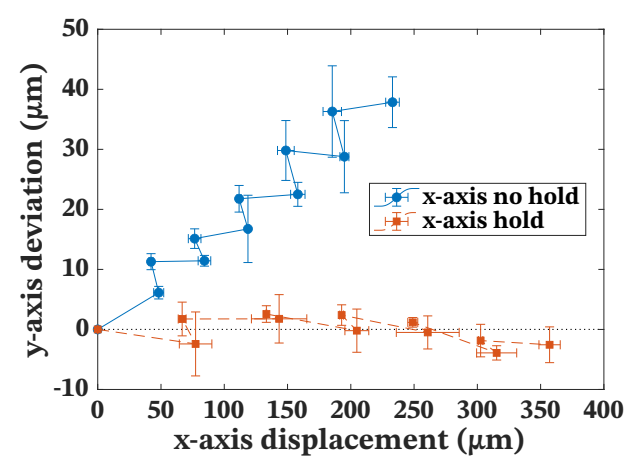

Figure 13. 1D displacements along the $x$ axis.

From Figure 13, the holding current improved the straightness of the axial displacement. The cumulative $y$-axis deviation without holding current was $37.8 \mu \mathrm{m}$ whereas with holding current was $2.55 \mu \mathrm{m}$. The deviation was dominant towards $+y$-axis without holding current, whereas it had no tendency with the holding current; this deviation could be caused by a tilt error of the DAA. We observed that the holding current minimizes this tilt error on the executed trajectories, easing the DAA's set-up.

\subsection{D Trajectory Validation}

To evaluate the bi-dimensional trajectory control of the DAA, a $0.416 \mathrm{~g}$ object, with size $20 \mathrm{~mm} \times 20 \mathrm{~mm}$ (the size of a $2 \times 2 \mathrm{DAA}$ ), was conveyed. Five target points were given to the $\mathrm{A}^{*}$ trajectory control algorithm using a holding current of $1 \mathrm{~A}:(0,0) ;(0,-259) ;(296,-259)$; $(296,0)$ and $(0,0)$, all in $\mu \mathrm{m}$. These points formed a rectangular objective trajectory. The trajectory control algorithm generated the control currents and injected them into the DAA to convey the object. The initial and final position measures of each conveyance step were taken using the Grasshopper camera. The initial position of each conveyance step was defined as the position before the displacement step and the final position as the position after the return phase of the DAA. Once the rectangular trajectory was performed, the system was reset and the same trajectory executed. This process was done 8 times to obtain a mean position for each conveyance step with its standard deviation in the $x$ and $y$ axes.

Figure 14 presents the objective rectangular trajectory with its target positions and predicted intermediate positions (noted "model") and the average of the experimental measurements. The standard deviation of the experimental results were small, indicating a repeatable outcome of the system. The DAA had a tendency to deviate towards the $+x$-axis while executing displacement steps on the $y$-axis. This could be the influence of the tilt error previously explained. There was a deviation towards the $-y$-axis while executing displacement steps along the $-x$-axis, but when executing displacement steps along the $+x$-axis there was no deviation tendency (apart from the correction step to attain the $(296,-259)$ point). This indicated that the flatness condition only affected one axis. Studying the displacement values, the system performed: larger-than-expected steps along the $y$-axis (both negative and positive); shorter-than expected steps along the $+x$-axis; and well-expected steps along the $-x$-axis. The final mean experimental position was $(-12.56 \mu \mathrm{m},-15.52 \mu \mathrm{m}) \pm 12.75 \mu \mathrm{m}$ for the $x$-axis and $2.2 \mu \mathrm{m}$ for the $y$-axis. As the wished final position was $(0,0)$, these values are also the final position error. 


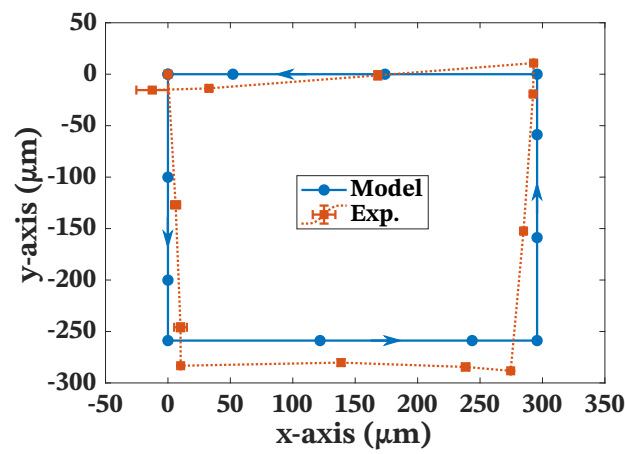

(a)

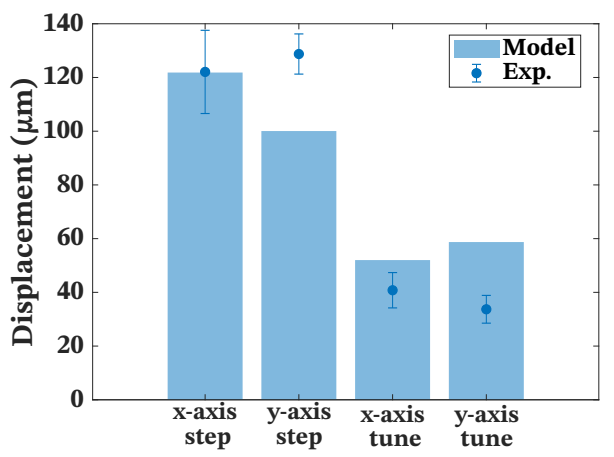

(b)

Figure 14. (a) 2D displacement of the conveyed object aiming the points $(0,-258.8),(295.7,-258.8)$, $(295.7,0),(0,0)$ [values in $\mu \mathrm{m}$ ] following the developed $A^{*}$ trajectory algorithm result. (b) Theoreticalexperimental comparison for the rectangular trajectory decomposed in displacement step and correction steps for each axis.

The rectangular trajectory is divided on its component displacement steps: eight normal displacement step (four along the $x$-axis plus four along the $y$-axis) and four tuning or adjusting steps (two along the $x$-axis plus two along the $y$-axis). The tuning steps come from the trajectory control algorithm with $\mathrm{A}^{*}$ : it executes the largest possible step in the wished direction, the maximum possible number of times and, once the object arrives near the wished position, the controller adjusts the last step to reach the position. Figure $14 \mathrm{~b}$ compares the model-predicted displacement for each type of step with the corresponding experimental results. From Figure 14b, the $x$-axis displacement step was almost perfectly predicted (122.1 $\mu \mathrm{m}$ exp. against $121.1 \mu \mathrm{m}$ model). For the $y$-axis step, the experimental displacement was larger than expected $(128.7 \mu \mathrm{m}$ exp. against $100.1 \mu \mathrm{m}$ model). The root-mean-square error for the four type of steps was $19.8 \mu \mathrm{m}$.

Finally, to evaluate longer and more complex trajectories, the trajectory controller was given a predefined trajectory forming the Université de Technologie de Compiègne acronym (UTC) (Figure 15). The trajectory spanned a $1700 \mu \mathrm{m} \times 800 \mu \mathrm{m}$ area and was $1109 \mu \mathrm{m}$ long. The $\mathrm{A}^{*}$ trajectory algorithm and $1 \mathrm{~A}$ holding current controlled the DAA.

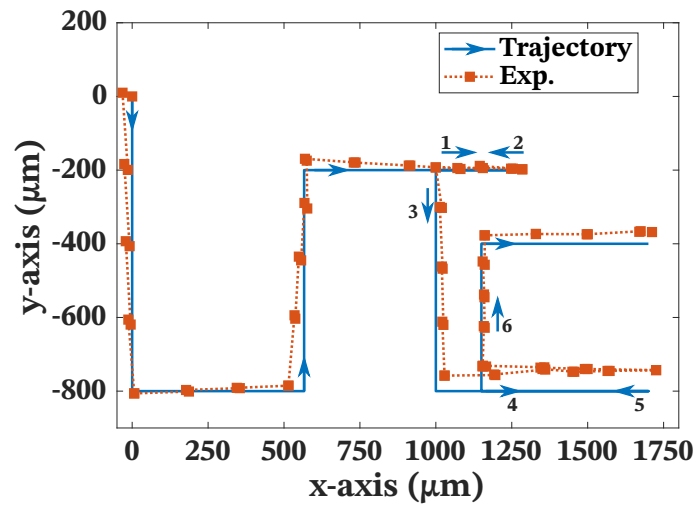

Figure 15. Bi-dimensional trajectory forming the Universite de Technologie de Compiegne acronym on a $2 \times 2$ DAA.

From Figure 15, the trajectory was well executed, with a maximum position error of $50.4 \mu \mathrm{m}$ for the $x$-axis and $67.5 \mu \mathrm{m}$ for the $y$-axis. The final position error was $11.8 \mu \mathrm{m}$ for the $x$-axis and $31.8 \mu \mathrm{m}$ for the $y$-axis.

The tests in this section demonstrate the DAA can execute guided planar motions (trajectories) thanks to the adapted $\mathrm{A}^{*}$ pathfinding algorithm and the developed feedforward control strategy. 


\section{Conclusions and Perspectives}

This paper presented a planar motion micro-conveyor based on a digital electromagnetic actuators array. The developed dynamic model of the system adequately predicted the system behavior for all current inputs with low root-mean-square errors. Based on experimental tests, a working zone with high driving currents was identified for small, repeatable and precise object displacement steps $(26 \mu \mathrm{m}$ displacements with $2 \mu \mathrm{m}$ straightness error). During experimental characterization, it was observed that the holding current decreased the straightness error (from a mean of $13 \mu \mathrm{m}$ without holding current to $4 \mu \mathrm{m}$ with $1 \mathrm{~A}$ holding current). During conveyance, an experimental return displacement was observed, attributed to possible uneven distribution of the conveyed object weight on the elementary actuators and heterogeneity in friction conditions. The return current intensity influence on this return step was studied and an operation point was determined. The ability of the proposed device to realize open-loop planar motions was shown with a displacement root-mean-square-error of $19.8 \mu \mathrm{m}$ for a $1109 \mu \mathrm{m}$-long trajectory. The work presented in this article demonstrated that the proposed device can execute guided planar motions (trajectories) thanks to the adapted $\mathrm{A}^{*}$ pathfinding algorithm and the developed feed-forward control strategy.

A comparison of our system against other micro-conveyor solutions found in the literature is presented in Table 2. Based on this comparison, the main interests of the proposed system are the digital principle, which enables a simple control, a high scalability and the possibility to obtain planar conveyance tasks. In terms of performances, the system presents competitive conveyance speed, conveyed object mass capability, resolution and precision compared to other systems. This comparison demonstrates then that the proposed system represents a viable alternative for conveyance systems in micro-factory.

Future work will focus on long range planar motions based on a new digital actuator array design composed of assembled elementary modules to obtain a larger motion area. A control of this larger conveyance area will be developed considering several conveyed objects with the objective to determine the optimal trajectories while avoiding collisions. With the objective to keep a simple open-loop control, we will explore the benefit of a time to time measurement of the conveyed object position in order to readjust the trajectory in case of large number of displacement steps. The proposed dynamic model will be also extended in order to simulate complex planar motions, including object rotations.

Table 2. Literature comparison.

\begin{tabular}{|c|c|c|c|c|c|c|c|c|c|c|}
\hline Ref. & Contact & Array & DoF & A.P & Control & Surface $(\mathrm{mm})$ & Object (mm) & $\begin{array}{c}\text { Speed } \\
\left(\mathrm{mm} \mathrm{s}^{-1}\right)\end{array}$ & $\begin{array}{l}\text { Resolution } \\
\quad(\mu \mathrm{m})\end{array}$ & $\begin{array}{c}\text { Precision } \\
(\mu \mathrm{m})\end{array}$ \\
\hline \multicolumn{11}{|c|}{ Electrostatic conveyors } \\
\hline [6] & Yes & Yes & 2 & Analog & Open & $264 \times 264$ & $132 \times 132,11 \mathrm{~g}$ & 354 & 400 & - \\
\hline \multicolumn{11}{|c|}{ Electrothermal conveyors } \\
\hline [20] & Yes & Yes & 3 & Digital & Both & $40 \times 40$ & $5 \times 7,24 \mathrm{mg}$ & 0.67 & 2 & 2 \\
\hline [21] & Yes & Yes & 3 & Digital & Both & $16 \times 16$ & $0.8 \times 0.8,78 \mu \mathrm{g}$ & 0.057 & 0.28 & 1 \\
\hline \multicolumn{11}{|c|}{ Piezoelectric conveyors } \\
\hline [9] & No & Yes & 2 & Analog & Closed & $10 \times 10$ & $300,129 \mathrm{~g}$ & 0.3 & 2 & 0.07 \\
\hline \multicolumn{11}{|c|}{ Pneumatic conveyors } \\
\hline [10] & No & Yes & 3 & Analog & Closed & $75 \times 75$ & 150 & 300 & - & 93 \\
\hline [11] & No & No & 3 & Analog & Closed & $228 \times 204$ & - & 80 & 200 & - \\
\hline [12] & Yes & Yes & 16 & Analog & Closed & $110 \times 93$ & - & 70 & - & - \\
\hline \multicolumn{11}{|c|}{ Electromagnetic conveyors } \\
\hline [7] & No & No & 3 & Analog & Open & $4 \times 4$ & - & - & - & - \\
\hline [8] & No & No & 3 & Analog & Closed & $8 \times 8$ & - & 2.3 & 100 & 10 \\
\hline \multicolumn{11}{|c|}{ Presented digital actuator array } \\
\hline Present & Yes & Yes & 3 & Digital & Open & $100 \times 100$ & $8.9 \mathrm{~g}$ & 2 & 8 & 5 \\
\hline
\end{tabular}


Supplementary Materials: The following are available online at https:/ / www.mdpi.com/article/10 .3390 /app112411980/s1, Video S1: MPM switching (driving current of 3.5 A) and the plate displacement (two steps) along $x$-axis.

Author Contributions: Conceptualization, S.D.T., L.P. and C.P.; methodology, S.D.T.; software, S.D.T. and A.T.; formal analysis, S.D.T.; investigation, S.D.T. and A.T.; writing-original draft preparation, S.D.T. and A.T.; writing-review and editing, S.D.T., L.P. and C.P.; visualization, S.D.T. and A.T.; supervision, L.P.; project administration, S.D.T., L.P. and C.P.; funding acquisition, L.P. All authors have read and agreed to the published version of the manuscript.

Funding: This work was funded by a public grant overseen by the French National Research Agency (ANR) as part of the "Tridimensional micro-conveyance systems for micro-factory" program (ANR15-CE10-0002-01)

Institutional Review Board Statement: Not applicable.

Informed Consent Statement: Not applicable.

Conflicts of Interest: The authors declare no conflict of interest. The funders had no role in the design of the study; in the collection, analyses, or interpretation of data; in the writing of the manuscript, or in the decision to publish the results.
Abbreviations
EDA elementary digital actuator
DAA digital actuator array
DoF degree of freedom
FPM fixed permanent magnet
MAE mean-absolute error
MPM mobile permanent magnet
RMSE root-mean-square error

The following abbreviations are used in this manuscript:

\section{References}

1. Hsu, T.R. Miniaturization. A paradigm shift in advanced manufacturing and education. In Proceedings of the 2002 IEEE/ASME International Conference on Advanced Manufacturing Technologies and Education in the 21st Century, Chia-Yi , China, 10-15 August 2002; pp. 1-19.

2. Mishima, N.; Tanikawa, T.; Ashida, K.; Maekawa, H. Design of a Microfactory. In Proceedings of the ASME 2002 Design Engineering Technical Conferences and Computer and Information Engineering Conference, Montreal, QC, Canada, 29 September2 October 2002.

3. Okazaki, Y.; Mishima, N.; Ashida, K. Microfactory-Concept, History, and Developments. J. Manuf. Sci. Eng. 2004, $126,837$. [CrossRef]

4. Järvenpää, E.; Heikkilä, R.; Siltala, N.; Prusi, T.; Tuokko, R. Micro-factories. In Micromanufacturing Engineering and Technology: Second Edition, 2nd ed.; Qin, Y., Ed.; William Andrew Publishing: Norwich, NY, USA, 2015; Chapter 23, pp. 549-579. [CrossRef]

5. Zhakypov, Z.; Uzunovic, T.; Nergiz, A.O.; Baran, E.A.; Golubovic, E.; Sabanovic, A. Modular and reconfigurable desktop microfactory for high precision manufacturing. Int. J. Adv. Manuf. Technol. 2017, 90, 3749-3759. [CrossRef]

6. Hosobata, T.; Yamamoto, A.; Higuchi, T. Transparent Synchronous Electrostatic Actuator for Long-Stroke Planar Motion. IEEE/ASME Trans. Mechatronics 2015, 20, 1765-1776. [CrossRef]

7. Zhang, H.; Kou, B.; Zhang, H.; Jin, Y. A three-degree-of-freedom short-stroke lorentz-force-driven planar motor using a halbach permanent-magnet array with unequal thickness. IEEE Trans. Ind. Electron. 2015, 62, 3640-3650. [CrossRef]

8. Chen, M.Y.; Tsai, C.F.; Fu, L.C. A Novel Design and Control to Improve Positioning Precision and Robustness for a Planar Maglev System. IEEE Trans. Ind. Electron. 2019, 66, 4860-4869. [CrossRef]

9. Deng, J.; Liu, Y.; Liu, J.; Xu, D.; Wang, Y. Development of a planar piezoelectric actuator using bending-bending hybrid transducers. IEEE Trans. Ind. Electron. 2019, 66, 6141-6149. [CrossRef]

10. Guelpa, V.; Laurent, G.J.; Dahroug, B.; Le Fort-Piat, N. Modular Contact-Free Conveyors for Handling Planar Fragile Objects. IEEE Trans. Robot. 2017, 33, 92-101. [CrossRef]

11. Chen, X.; Zhong, W.; Li, C.; Fang, J.; Liu, F. Development of a contactless air conveyor system for transporting and positioning planar objects. Micromachines 2018, 9, 487. [CrossRef] [PubMed]

12. Robertson, M.A.; Murakami, M.; Felt, W.; Paik, J. A Compact modular soft surface with reconfigurable shape and stiffness. IEEE/ASME Trans. Mechatronics 2019, 24, 16-24. [CrossRef] 
13. Miao, X.; Dai, X.; Huang, Y.; Ding, G.; Zhao, X. Large out-of-plane displacement bistable electromagnetic microswitch on a single wafer. Sensors 2016, 16, 634. [CrossRef] [PubMed]

14. Dorbaum, M.; Tappe, S.; Ortmaier, T.; Ponick, B. Design and Analysis of Electromagnetic Tilting Actuators. IEEE/ASME Trans. Mechatronics 2019, 24, 2171-2181. [CrossRef]

15. Seok, T.J.; Quack, N.; Han, S.; Muller, R.S.; Wu, M.C. Highly Scalable Digital Silicon Photonic MEMS Switches. J. Light. Technol. 2016, 34, 365-371. [CrossRef]

16. Phung, H.; Nguyen, C.T.; Jung, H.; Nguyen, T.D.; Choi, H.R. Bidirectional tactile display driven by electrostatic dielectric elastomer actuator. Smart Mater. Struct. 2020, 29, 035007. [CrossRef]

17. Olbrich, M.; Schütz, A.; Bechtold, T.; Ament, C. Design and optimal control of a multistable, cooperative microactuator. Actuators 2021, 10, 183. [CrossRef]

18. Schmitt, L.; Schmitt, P.; Hoffmann, M. 3-Bit digital-to-analog converter with mechanical amplifier for binary encoded large displacements. Actuators 2021, 10, 182. [CrossRef]

19. Baishya, N.J.; Bhattacharya, B.; Ogai, H.; Tatsumi, K. Design of an Anti-Slip Mechanism for Wheels of Step Climbing Robots. Actuators 2021, 10, 259. [CrossRef]

20. Ataka, M.; Legrand, B.; Buchaillot, L.; Collard, D.; Fujita, H. Design, fabrication, and operation of two-dimensional conveyance system with ciliary actuator arrays. IEEE/ASME Trans. Mechatronics 2009, 14, 119-125. [CrossRef]

21. Ellerington, N. 3 DoF, Long Range Planar Lift and Slice Micro-Conveyor with Vision-Based Control System. Ph.D. Thesis, Dalhousie University, Halifax, Nova Scotia, 2012. [CrossRef]

22. Duque Tisnes, S.; Petit, L.; Prelle, C.; Lamarque, F. Modeling and experimental validation of a planar micro conveyor based on a $2 \times 2$ array of digital electromagnetic actuators. IEEE/ASME Trans. Mechatronics 2021, 26, 1422-1432. [CrossRef]

23. Furlani, E.P. Permanent Magnet Furthermore, Electromagnetical Devices; Elsevier: Rochester, NY, USA, 2001 ; p. 537.

24. Bengisu, M.T.; Akay, A. Stability of friction-induced vibrations in multi-degree-of-freedom systems. J. Sound Vib. 1994, 171, 557-570. [CrossRef]

25. Marques, F.; Flores, P.; Pimenta Claro, J.C.; Lankarani, H.M. A survey and comparison of several friction force models for dynamic analysis of multibody mechanical systems. Nonlinear Dyn. 2016, 86, 1407-1443. [CrossRef]

26. Semat, H.; Katz, R. Physics Chapter 10: Momentum and Impulse. In Physics; Robert Katz Publications; DigitalCommons University of Nebraska-Lincoln: New York, NY, USA, 1958; Chapter 10, p. 142.

27. Weir, G.; Tallon, S. The coefficient of restitution for normal incident, low velocity particle impacts. Chem. Eng. Sci. 2005, 60, 3637-3647. [CrossRef]

28. Duque Tisnés, S.; Petit, L.; Prelle, C. Control strategies for a micro-conveyance system based on an electromagnetic digital actuator array. In Proceedings of the 12th France-Japan Congress, 10th European-Asia Congress in Mecatronics, Tsu, Japan, 10-12 September 2018. [CrossRef] 